\title{
Experiencias de extensionismo y capacitación sobre manejo y conservación de manglares en dos comunidades rurales en artemisa, Cuba
}

DOI: $\underline{\text { https://doi.org/10.33262/ap.v3i3.2.95 }}$

\begin{abstract}
(c) (6)(2) (2)
Experiences of extensionism and training on management and conservation of mangroves in two rural communities in Artemisa, Cuba
\end{abstract}

Yanet Castillo Arrebato. ${ }^{1}$, Yudemir Cruz Pérez. ${ }^{2}$, Ángel Zaldívar Solís. ${ }^{3}$ \& Luis Efraín Velastegui López. ${ }^{4}$

\begin{abstract}
The purpose of this work was to value experiences training extensionists on handling of the swamp, located in the El Mambí and Loma Colorada communities of the municipality San Cristóbal, Artemisa. The investigation was developed in three stages: the participative diagnostic, the design, execution and evaluation of a training plan, the pursuit and sustainability of the actions training extensionists. They were used technical and tools of the diagnostics rural participative and of the forest extension as they were: you interview, work in group, participant and technical observations of hierarchization and consent; based on the visualization principles, triangulation and good ignorance. The results threw that among the community ones the positive attitudes prevailed in the boarding of the problem of the development, protection and conservation of the swamp; that it indicated to the own town like the favorable scenario to receive training in day schedules and with varied forms of popular instruction. The resources with smaller readiness and quality were the water and the drinkable water; and with regard to the arboreal vegetable species the promissory ones prevailed from their silvic, forestry,

\footnotetext{
${ }^{1}$ Unidad Empresarial de Base Agroindustrial Forestal San Cristóbal, Empresa Agroforestal Costa Sur, Cuba. Email: ycastillo80@ nauta.cu Móvil: (+53) 53714799.

2 Facultad de Turismo, Universidad de La Habana, Cuba. Emails: yudemir.cruz@ftur.uh.cu y cruzperezyudemir@gmail.com Móvil: (+53) 58151302.

${ }^{3}$ Facultad de Ciencias Forestales y Agropecuarias, Universidad de Pinar del Río "Hermanos Saiz Montes de Oca”, Cuba. Email: azaldivar@upr.edu.cu Telf.: (+53) 48779663

${ }^{4}$ Ciencia Digital Editorial, Ecuador, luisefrainvelastegui@cienciadigital.org
} 
economy and administration for the development of the forest component. The training plan, allowed the learning of lessons with a level of appropriate knowledge on the handling of the vegetable formation of Swamp, as well as the application of the legal mark; what will allow a positive movement of the local forest culture. The persistent training necessities in the community ones will allow the long-term work to give sostenibility to the actions extensionistic at local level.

Keywords: forest extension, local training, handling, swamp.

\section{Resumen}

El propósito de este trabajo fue valorar experiencias de extensionismo y capacitación sobre manejo y conservación del manglar, ubicado en las comunidades El Mambí y Loma Colorada del municipio San Cristóbal, Artemisa. La investigación se desarrolló en tres etapas: el diagnóstico participativo, el diseño, ejecución y evaluación de un plan de capacitación, el seguimiento y sostenibilidad de las acciones extensionistas de capacitación. Se utilizaron técnicas y herramientas del diagnóstico rural participativo y de la extensión forestal como fueron: entrevistas, trabajo en grupo, observaciones participantes y técnicas de jerarquización y consenso; basados en los principios de visualización, triangulación e ignorancia óptima. Los resultados arrojaron que entre los comunitarios predominaron las actitudes positivas en el abordaje del problema del fomento, protección y conservación del manglar; que indicó a la propia localidad como el escenario propicio para recibir capacitación en horarios diurnos y con formas variadas de instrucción popular. Los recursos con menor disponibilidad y calidad fueron el agua y el agua potable; y con respecto a las especies vegetales arbóreas predominaron las promisorias desde su sílvica, silvicultura, economía y gestión para el desarrollo del componente forestal. El plan de capacitación, permitió el aprendizaje de lecciones con un nivel de conocimiento adecuado sobre el manejo de la formación vegetal de Manglar, así como la aplicación del marco legal; lo que permitirá un movimiento positivo de la cultura forestal local. Las necesidades de capacitación persistentes en los comunitarios permitirán el trabajo a largo plazo para darle sostenibilidad a las acciones extensionistas a nivel local.

Palabras clave: extensión forestal, capacitación local, manejo, manglar

\section{Introducción}

Es bien conocida la importancia de los bosques de mangles y el papel que tienen a lo largo y ancho de las zonas tropical y subtropical del planeta en todo lo referente al manejo de zonas costeras, lo cual se dimensiona en estos tiempos, en que el Cambio Climático es una realidad concreta, y la elevación del nivel medio del mar una de sus consecuencias obviamente relacionadas con los manglares; aspectos con los que coinciden Lescarro et al. (2018). 
El desconocimiento de la importancia de los servicios ecosistémicos que brinda el manglar ha provocado cambios tanto en su estructura como en su funcionamiento, con consecuencias en su salud, lo que se traduce en perjuicios para los seres humanos (Menéndez, 2013), es por ello que el abordaje extensionista de la actividad de manejo y protección de los manglares, resulta una actividad de interés sociocultural, ambiental, económico y para el desarrollo a nivel local.

Entre las formas más conocidas de intervención sociocultural en materia agrícola y forestal se encuentra el proceso extensionista (Camargo, Páez y Fonseca, 2019), visto desde su dimensión más amplia e integradora y no desde una concepción reduccionista de asistencia técnica, sino con el énfasis en la educación de la gente, su organización y desarrollo integral (Ramsay y Beltrán, 2007). Por lo que resulta bastante adecuada la práctica de la extensión agraria para el abordaje de los problemas locales en consonancia con los criterios de Sousa et al. (2020).

Sobre la base de lo expuesto anteriormente, este trabajo pretende realizar un modesto aporte al uso de la extensión forestal para el abordaje del problema del manejo y conservación de los manglares, los cuales han recibido el impacto de disímiles eventos de origen antrópico y natural en la provincia de Artemisa en los últimos años (Departamento Meteorológico, Citma, 2017), debido a los períodos prolongados de intensas lluvias o escasas precipitaciones; y específicamente en localidades costeras del municipio de San Cristóbal de la provincia de Artemisa en Cuba; todo lo cual aportará experiencias para el futuro trabajo en materia forestal con las comunidades rurales del territorio.

\section{Materiales Y Métodos}

\section{Descripción físico - geográfica del área de estudio}

La zona costera del municipio San Cristóbal está localizada en la zona suroccidental del país, en la Llanura Sur-Occidental de la Provincia Artemisa (llanura costera muy baja), incluye los Consejos Populares costeros: José Martí (que limita al Este con el municipio Candelaria, y abarca desde la desembocadura arroyo "Los Colorados" hasta la desembocadura río San Cristóbal), y Ramón López Peña (que limita al Oeste con el municipio Los Palacios, provincia de Pinar del Río, y abarca desde la desembocadura del río Bacunagua hasta la desembocadura río "San Cristóbal”).

Comprende un extenso territorio de $28.94 \mathrm{Km}$ de línea de costa, abarca una superficie total de 15693.91 ha, de ellas corresponde 6169.00 ha a tierras agrícolas, 9080.71 ha de manglar, 12.76 ha de manigua costera, 408.63 hade lagunas temporales y permanentes, 22.81 ha de los asentamientos El Mambí y poblado Loma Colorada).

La zona se halla expuesta a múltiples peligros de origen natural. Pero sin dudas, los hidrometeorológicos son causantes de los mayores desastres acaecidos en su entorno, asociados fundamentalmente al azote de los ciclones tropicales. En ello inciden la morfología y posición geográfica del territorio, que hacen que este se "interponga" en la 
trayectoria de una parte significativa de los huracanes que se forman en las cálidas aguas del Mar Caribe y del Atlántico Sur, y que circulan hacia el Golfo de México.

Se suma a esto el hecho de que cada día es más notoria la vulnerabilidad de los asentamientos humanos ante los eventos climatológicos extremos, lo cual ha suscitado grandes afectaciones socioeconómicas y ambientales a su paso, siendo el territorio de la provincia Artemisa, sin dudas, uno de los más perjudicados de todo el país, las afectaciones tras el paso de los huracanes Gustav e Ike en el año 2008, fueron significativas.

\section{Trayectoria metodológica de la investigación en campo}

En estas comunidades de El Mambí y Loma Colorada, asentamientos rurales costeros, se realizó el diagnóstico participativo, según Expósito (2003) y Ramírez y Camacho (2019). Para determinar la situación actual de la cultura forestal comunitaria, basada en métodos y herramientas del diagnóstico rural participativo y de la extensión forestal sugeridos por Cárdenas et al. (2006).

La causa del diagnóstico fue: la necesidad del cambio, y este se desarrolló siguiendo un triple enfoque, en el que se analizó la realidad objetiva: todo lo que rodeaba a la localidad en bienes y servicios (personas, masa boscosa, recursos forestales, suelos, agua...), la realidad subjetiva: basada en el pensamiento de las personas según sus conocimientos, actitudes y preferencias, y la práctica: lo que los hombres y mujeres fueron capaces de realizar en función de la transformación de su realidad. El diagnóstico también siguió los principios de visualización, triangulación e ignorancia óptima.

Se aplicaron dos entrevistas (Muñoz, 2015), una semiestandarizada cuyo objetivo fue captar información respecto a la frecuencia, intensidad y consecuencias con que ocurren las inundaciones costeras; y una estandarizada con el objetivo de determinar el conocimiento, la actitud y las preferencias respecto a la capacitación sobre el manejo y conservación de los manglares; así como lo relativo a lo que la Ley Forestal expresa al respecto.

Después del diagnóstico participativo se planificó la capacitación considerando la cantidad del público objetivo y sus características (grupos etarios, composición por sexo, oficios, intereses...), lo que permitió definir las formas de capacitación y los facilitadores locales y externos que podían ayudar al mejor desarrollo de este.

Se diagnosticaron las necesidades de capacitación persistentes y de elevación de nivel a través de una lista de qué quedó y qué quieren aprender. En este proceso se repitió la priorización de necesidades, en vez de las necesidades que quedaron en función de darle sostenibilidad a la capacitación.

\section{Resultados y Discusión}

Se realizaron recorridos en el sistema manglar-ciénaga acompañando a los diferentes usuarios para observar y describir en detalle las actividades que ahí realizan, así como 
otro tipo de información (opiniones, comentarios, entre otros...) que pudieran servir para compararla con otras fuentes.

En el municipio de San Cristóbal existe una tendencia al incremento de factores degradantes de los manglares, aunque este no se encuentra entre los municipios con mayor incidencia anual; pero en las localidades de El Mambí y Loma Colorada, entre estos factores predominan los de origen antrópico (A) y los de origen natural $(\mathrm{N})$, todos cada vez más frecuentes, intensos y con disímiles perjuicios a la comunidad; lo que pudo ser constatado en la praxis durante el diagnóstico, como:

(A). Quemas no controladas.

(A). Incremento de las fronteras de las parcelas agropecuarias.

(A). Producción ilegal de carbón vegetal de especies del manglar.

(A). Tala ilegal y extracción de leña y corteza.

(A). Pesca furtiva de especies del ecosistema.

(A). Contaminación localizada, con combustibles y lubricantes de embarcaciones pesqueras.

(A). Incultura ambiental (vertimientos de residuos líquidos y sólidos de los comunitarios, proliferación de basureros...).

(N). Impacto de fenómenos hidrometeorológicos extremos (principalmente ciclones tropicales, sequías extremas, inundaciones por intensas lluvias y fuertes vientos).

(N). Elevación paulatina del nivel del mar y otros efectos del cambio climático global, regional y local.

(N). Incremento de la ocurrencia e intensidad de incendios forestales y/o rurales, de origen natural.

(N). Cambios en las etapas sucesiones del manglar y su fenología, espontáneos o generados por otros factores naturales.

\section{- Conocimientos, actitudes y preferencias para la capacitación}

Una vez sondeada la percepción del problema se determinaron los niveles de conocimientos, actitudes y preferencias comunitarias acerca de los manejos que se le realizan a los manglares y los aspectos sobre la capacitación en este tema, resultando de la entrevista estandarizada (Fig. 1) que los comunitarios presentaron un nivel de conocimiento predominantemente bajo relativo al problema; que los hombres en ambas comunidades tuvieron niveles de conocimiento más bajos que las mujeres, lo que se contrapone al hecho de que sean los varones los que ostenten mayores niveles de instrucción o grado de escolaridad. 


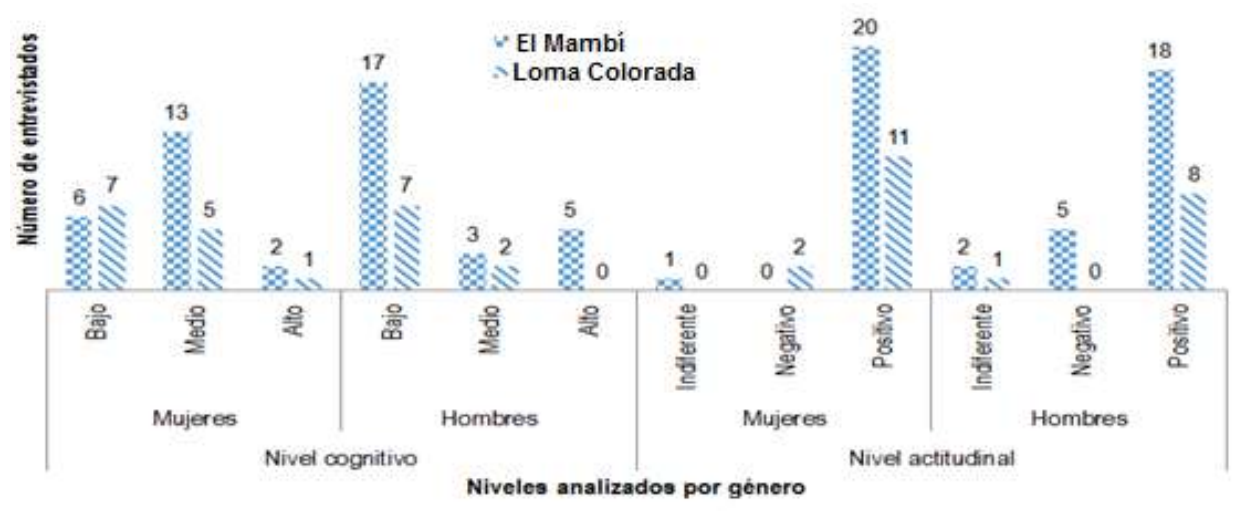

Figura 1. Conocimientos y actitudes de los comunitarios.

Fuente: Elaboración propia.

Sin embargo, este resultado pudo estar influenciado por la necesidad que han tenido los hombres con el uso indebido del manglar, y las respuestas dadas no hayan sido veraces o estén manifestando un temor al enfrentamiento con su actuar. Karremans (2004), aborda que el comportamiento del hombre "rural" puede estar afectado durante investigaciones y participaciones de especialistas externos a las comunidades.

Con respecto a las actitudes, en ambas comunidades y para ambos géneros, prevalecieron las personas con actitudes positivas en el abordaje de la conservación de los manglares como problema que afecta a su localidad. Por lo que se puede inferir que el nivel cognitivo no fue importante para la identificación y tratamiento social de los manglares, aunque la cognición se haga necesaria para la gestión de la cultura forestal.

\section{- Descripción de las experiencias de extensionismo y capacitación}

Las experiencias de extensionismo y capacitación se validaron, en primer lugar, con el cumplimiento formal de las actividades planificadas en un ciento por ciento; con una participación promedio por actividad de 27 comunitarios, donde la comunidad de El Mambí presentó la más estable participación en número y repetición de personas.

La relación promedio de género por actividad fue de 2:1 (Hombres /Mujeres) entre ambas localidades, y de 1:1 para El mambí y 1:2 para Loma Colorada, lo que fortaleció el trabajo con el enfoque a ambos géneros en proporción con la población total en cada localidad.

En resumen (Tabla 1), puede expresarse que el cumplimiento formal de las actividades planificadas, tuvieron resultados positivos, lo que sirve de premisa para el continuo perfeccionamiento de la capacitación en materia de manejo y conservación de los manglares en ambas localidades.

Tabla 1. Principales resultados obtenidos con la realización de cada una de las cuatro actividades de capacitación, según las valoraciones del grupo en acción, cada día.

\begin{tabular}{cll}
\hline Actividad & \multicolumn{1}{c}{ Principales resultados } \\
\hline Charla & a. El grupo se conoció mejor y aceptaron más a los facilitadores de la \\
& $\begin{array}{l}\text { capacitación. } \\
\text { b. Se identificaron varios líderes. }\end{array}$ \\
\hline
\end{tabular}


Tabla 1. Principales resultados obtenidos con la realización de cada una de las cuatro actividades de capacitación, según las valoraciones del grupo en acción, cada día.

(continuación)

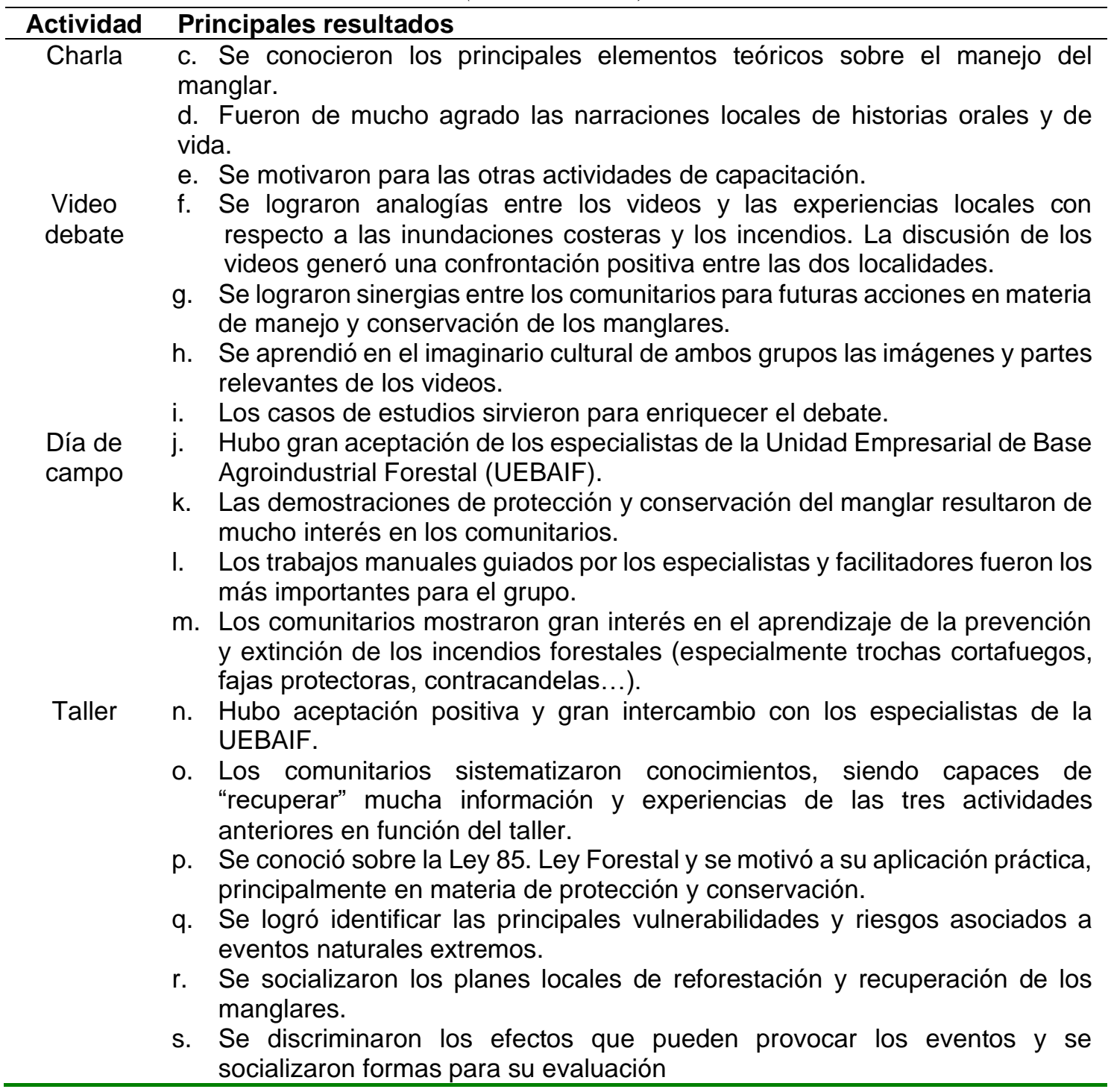

Fuente: Elaboración propia.

La sistematización del plan de capacitación desarrollado permitió listar las lecciones aprendidas por los comunitarios en cada localidad, por lo que de acuerdo con las aportaciones grupales se presentan las siguientes listas:

- Lecciones aprendidas por los comunitarios de El Mambí:

$\checkmark$ Las inundaciones costeras son producidas por eventos meteorológicos extremos, como intensas lluvias y fuertes vientos.

$\checkmark$ Los incendios forestales son aquellos que ocurren en áreas donde existe vegetación, excepto los prescritos por los especialistas del sector forestal.

$\checkmark$ La Ley 85/98 es la Ley Forestal y en ella se recogen deberes y derechos, entre los que se encuentran los referidos a la protección de los bosques, y especialmente los costeros. 
$\checkmark$ En la comunidad pueden darse alrededor de 18 situaciones peligrosas, por lo que se deben establecer las normas de seguridad principales para prevenir y combatir el fuego

$\checkmark \quad$ La forestal, encabezados por el guardia forestal y el jefe de área de la localidad deberán tener planes para enfrentar los peligros y las vulnerabilidades y riesgos de inundaciones e incendios.

$\checkmark$ Los eventos extremos pueden provocar pérdidas económicas, de vidas humanas, a los bienes personales y colectivos, así como daños al medio ambiente.

- Lecciones aprendidas por los comunitarios de Loma Colorada:

$\checkmark$ No podemos ser negligentes donde halla bosque o cerca de este.

$\checkmark$ El guardia forestal y el jefe de área son los encargados de prepararnos a todos para evitar afectaciones frente a eventos extremos.

$\checkmark$ La Ley Forestal en Cuba es la 85 del 98.

$\checkmark$ En la seca es donde más pueden ocurrir incendios.

$\checkmark$ La seca en nuestra localidad es de diciembre a abril.

$\checkmark$ Aprendimos de varios métodos para cultivar, proteger y conservar los manglares.

Las lecciones aprendidas dieron fundamento a los resultados obtenidos en el diagnóstico final, donde se expresaron los niveles cognitivos y de satisfacción logrados en los comunitarios.

\section{Conclusiones}

- Entre los comunitarios predominaron las personas con actitudes positivas en el abordaje del problema del manejo y protección de manglares, y el impacto de eventos frecuentes, intensos y con disímiles perjuicios locales; que indicaron a las propias localidades como el escenario propicio para recibir capacitación en horarios diurnos y con formas libres de los esquemas más tradicionales y ortodoxos de instrucción popular.

- En ambas localidades, los recursos valorados con menor disponibilidad y calidad fueron el agua y el agua potable; y con respecto a las especies vegetales arbóreas predominaron las promisorias desde su sílvica, silvicultura, economía y gestión para el desarrollo del componente forestal en planes futuros.

- En la práctica social el plan de capacitación, permitió el aprendizaje de lecciones con un nivel de conocimiento adecuado sobre los eventos extremos, la protección y conservación de los manglares, así como sobre la Ley Forestal y su aplicación; lo que permitirá un movimiento positivo de la cultura forestal local, de acuerdo con los niveles elevados de satisfacción de los comunitarios con respecto a la capacitación desarrollada.

- Las necesidades de capacitación persistentes en los comunitarios permitirán el trabajo a largo plazo a través de actividades planeadas que se orienten hacia el 
cambio de conocimientos, habilidades y aptitudes de las personas en el abordaje de los problemas, desde la autogestión y de forma participativa, para darle tratamiento a la sostenibilidad de las acciones extensionistas y lograr un desarrollo más justo y sustentable a nivel local.

\section{Bibliografía}

Camargo, E.; Páez, E. y Fonseca, J. (2019). Desarrollo y extensión rural: Estrategias para el fortalecimiento de la agricultura familiar campesina. Escuela de Ciencias Agrícolas, Pecuarias y del Medio Ambiente, Ed.: UNAD, Bogotá, Colombia. eISBN: 978-958-651-731-7.

Cárdenas, I., Caballero, R., Palenzuela, L., Martínez, M., Sáez, A. (2006). El extensionista. Promotor del Desarrollo Forestal Sostenible. Ediciones Unidad de Producciones Gráficas del MINREX, La Habana, Cuba. 76 p.

Departamento de Meteorología del CITMA (2017). Ciclones tropicales y huracanes que han afectado a Cuba y al Caribe. Disponible en: file:///D:/busque\%20para\%20maestrante/Ciclones\%20tropicales\%20y\%20hurac anes\%20que $\% 20$ han $\% 20$ azotado $\% 20 \mathrm{Cuba} \% 20 \mathrm{y} \% 20 \mathrm{el} \% 20 \mathrm{Caribe} . \mathrm{htm}$

Expósito, V. (2003). Diagnóstico rural participativo. Centro Cultural Poveda, Santo Domingo, República Dominicana, 118 p.

Karremans, J. (2004). Sociología y antropología para el desarrollo rural. CATIE. Turrialba, Costa Rica, 194 p.

Lescarro, E., Rojas, Á., Reales, P., Rocha, E., Sánchez, M. y Rizo, G. (2018). Comportamiento pro ambiental mediado por la investigación como estrategia pedagógica para la conservación de manglares. MODULO ARQUITECTURACUC, vol. 20, no. 1, pp. 61-70. DOI: 10.17981/mod.arq.cuc.20.1.2018.06

Menéndez, L. (2013). El ecosistema de manglar en el archipiélago cubano: bases para su gestión [en línea]. Tesis presentada en opción de grado Científico de Doctor en Ciencias. España: Universitat d'Alacant - Universidad de Alicante. [Consulta: 2 abril 2018]. Disponible en: https://dialnet.unirioja.es/servlet/tesis?codigo=59555

Muñoz, C. (2015). Metodología de la Investigación. Ciencias Sociales. Ed.: Progreso S.A de C.V, México D, F. 307p. ISBN 9786074265422

Ramírez, A. y Camacho, M. (2019). Diagnóstico participativo para determinar problemas ambientales en comunidades rurales. Revista Telos: 21(1), 86-113. ISSN: 13170570 .

Ramsay, J., Beltrán, L. 2007. Extensión agraria estrategia para el desarrollo rural. Instituto Interamericano de Cooperación para la Agricultura. Segunda Edición. República Bolivariana de Venezuela. 458 p. 
Sousa, L. M. R.; Conceição, A. K. C.; Lira, Á, G. S.; Maestri, M. P.; Aquino, M. G. C. Diagnóstico rural participativo da comunidade de Lavras, Santarém/PA, Amazônia. Natural Resources, v.10, n.2, p.44-48, 2020. DOI: http://doi.org/10.6008/CBPC2237-9290.2020.002.0006 


\section{PARA CITAR EL ARTÍCULO INDEXADO.}

Castillo Arrebato, Y. ., Cruz Pérez, Y., Zaldívar Solís, Ángel, \& Velastegui López, L. E. (2021). Experiencias de extensionismo y capacitación sobre manejo y conservación de manglares en dos comunidades rurales en artemisa, Cuba. AlfaPublicaciones, 3(3.2), 1929. https://doi.org/10.33262/ap.v3i3.2.95

\section{LC Ciencia}

El artículo que se publica es de exclusiva responsabilidad de los autores y no necesariamente reflejan el pensamiento de la Revista Alfa Publicaciones.

El artículo queda en propiedad de la revista y, por tanto, su publicación parcial y/o total en otro medio tiene que ser autorizado por el director de la Revista Alfa Publicaciones.
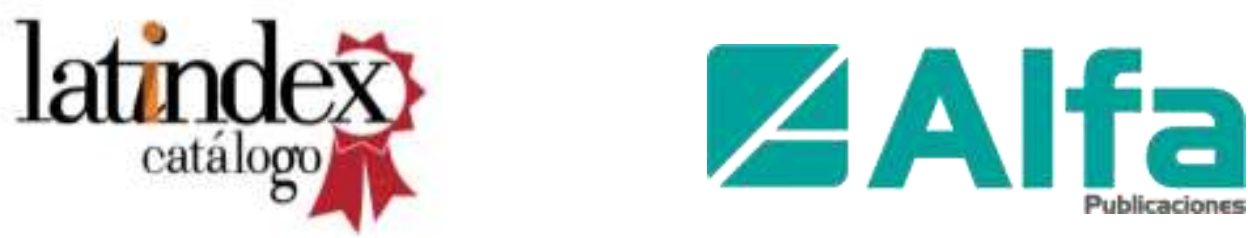